\title{
Defining Disease Progression and Drug Durability in Type 2 Diabetes Mellitus
}

\author{
Sanjay Kalra, ${ }^{1}$ Nor Azmi Kamaruddin, ${ }^{2}$ Jayanti Visvanathan, ${ }^{3}$ Ravi Santani ${ }^{4}$
}

1. Department of Endocrinology, Bharti Hopsital, Karnal, India; 2. Endocrine Unit, Department of Medicine, Universiti Kebangsaan Malaysia Medical Centre, Kuala Lumpur, Malaysia; 3. Medical Affairs, Novartis (Singapore) Pte Ltd, Singapore; 4. Medical Affairs, Novartis Corporation (M) Sdn Bhd,

Petaling Jaya, Malaysia

T his communication shares insights into the definition of disease progression and drug durability in type 2 diabetes. Disease progression may be defined as gradual worsening of beta-cell function, clinically observed as an increase in drug dosage, drug frequency or number of glucose lowering drugs needed to maintain $\mathrm{HbA} 1 \mathrm{c}$ control; and/or a $\geq 0.5 \%$ rise in $\mathrm{HbA} 1 \mathrm{C}$, unexplained by acute, modifiable factors, while using the same drug regimen; and/or as the occurrence or worsening of cardiovascular or microvascular complications, in spite of standard care, over a pre-specified time period. Durability of a drug or a drug combination may be defined as its ability to postpone or delay progression of disease, in a safe and well tolerated manner. Thus, all drugs that are able to prevent disease progression (i.e., postpone loss of glycaemic control, need for intensification of therapy or onset or worsening of complications) may be termed 'durable'.

\section{Keywords}

Combination therapy, drug failure, glycaemic control, monotherapy, type 2 diabetes

Disclosures: Jayanti Visvanathan is an employee of Novartis (Singapore) Pte Ltd. Ravi Santani is an employee of Novartis Corporation (M) Sdn Bhd. Nor Azmi Kamaruddin and Sanjay Kalra have no conflicts of interest to declare in relation to this publication.

Review Process: This article is a short opinion piece and has not been submitted to external peer reviewers, but was reviewed for accuracy by the editorial board before publication.

Compliance with Ethics: This article is an opinion piece and does not report on new clinical data, or any studies with human or animal subjects performed by any of the authors.

Authorship: The named author meets the Internationa Committee of Medical Journal Editors (ICMJE) criteria for authorship of this manuscript, take responsibility for the integrity of the work as a whole and have given final approval to the version to be published.

Received: 31 October 2018

Accepted: 15 December 2018

Citation: European Endocrinology. 2019;15(2):67-9

Corresponding Author: Sanjay Kalra, Department of Endocrinology, Bharti Hospital, Kunjpura Road, Karnal, 132001 India. E: brideknl@gmail.com

Support: No funding was received in the publication of this article.
The term 'disease progression' is ubiquitous in clinical medicine. It has been used in various medical specialties such as oncology, nephrology, dermatology and cardiology.1.-3 Type 2 diabetes (T2D), however, is a complex disease, which eludes simple definition of disease progression. The exhaustive list of pathophysiologic mechanisms that contribute to T2D is matched by an equally notable array of evidence-based drugs and drug combinations. ${ }^{4-6}$ These are backed by robust clinical trials with designs and objectives as diverse as T2D.? Therefore one may not necessarily find uniform definitions of concepts that are presumed to be commonly understood by other disciplines.

Two such constructs, which defy easy definition in T2D care, are the phrases 'disease progression' and 'drug durability'. In this editorial, we explore various definitions of diabetes progression and drug durability, before suggesting a comprehensively crafted description of both that should be acceptable to all.

\section{Defining disease progression - why is it important?}

Experts have long explored the various facets of disease progression and drug durability in T2D. The definition and characteristics of progression of T2D have been reviewed in detail. ${ }^{8}$ A stepwise approach to disease progression, including conversion from prediabetes to diabetes, the need for medication, loss of glycaemic control and occurrence of complications, has been proposed. ${ }^{8}$

A recent review of data identified the following risk factors for progression from prediabetes to diabetes: relatively higher fasting or postprandial glycaemia; a steeper rate of increasing fasting glucose; higher body mass index, blood pressure and triglycerides; and lower HDL cholesterol levels. ${ }^{9}$ Among people with T2D who had glycated haemoglobin (HDA1C) $<7 \%$ or no glucose lowering medications at baseline, predictors of diabetes progression ( $\mathrm{HbA} 1 \mathrm{C} \geq 7 \%$ or initiation of hypoglycaemic agent) include high baseline HbA1c, younger age, and weight gain. Each decade of increasing age reduces the progression risk by $15 \%$ and each $1 \mathrm{lb}(0.453 \mathrm{Kg})$ of increased weight is associated with $2 \%$ increased odds of progression..$^{10}$ The chances of requirement of glucose lowering medication among disease progressors, decreases by $40 \%$ with every decade of age, and decreases by $2.3 \%$ with each $1 \mathrm{mg} / \mathrm{dl}$ decrease from baseline LDL level. ${ }^{10}$ Loss of glycaemic control is a precursor of progression of T2D, and seems to be an integral part of the syndrome's natural history. However, not all patients lose glycaemic control at the same rate. According to the United Kingdom Prospective Diabetes Study (UKPDS), higher sulfonylurea failure rates are noted in individuals who are younger, have lower body weight, higher glucose concentrations, lower $\beta$-cell reserve and those randomised to glibenclamide rather than chlorpropamide.11

Drug durability has conventionally been studied from a limited, monotherapeutic perspective. A Diabetes Outcome Progression Trial (ADOPT), which compared failure rates of various drugs, 
reported a monotherapy failure of $15 \%$ with rosiglitazone, $21 \%$ with metformin and $34 \%$ with glyburide, at 5 years of therapy. ${ }^{12}$ In recent years, however, our approach to the management of T2D has changed. Earlier use of combination therapy is indicated, using drugs with a complementary mode of action. ${ }^{13}$ This evolution in therapy calls for a reassessment of the definition and scope of disease progression, as well as drug durability. This need is made more important by the fact that T2D now occurs in younger adults, who have a longer life expectancy to live with diabetes. ${ }^{14}$

\section{The characteristics of diabetes and methods for measuring its progression}

The natural history of $\mathrm{T} 2 \mathrm{D}$ is marked by a gradual decline in betacell secretory function; insulin sensitivity, on the other hand, remains constant, and shows no such decline. Disease progression, therefore, can be defined in terms of degree and rate of beta-cell secretory defect, or beta-cell failure. ${ }^{15}$ Both anatomical (beta-cell apoptosis) and functional (insulin secretory defect) markers have been used by experts to measure the rate of beta-cell failure. However, a combined anatomic-functional view of beta-cell health, appears the best approach. The three-stage model which lists beta-cell sufficiency, partial/reversible insufficiency, and complete/irreversible insufficiency, also appears a rational way of describing disease progression in T2D. ${ }^{16}$ Objective markers are needed, however, and fasting and stimulated serum C-peptide are useful markers for functioning of beta cells. This has minimal relevance in clinical practice, and is used primarily in research settings. Such a definition of disease progression has been used in trials in type 1 diabetes prevention. ${ }^{17}$ For T2D, the coefficient of failure, a simple tool developed by Wallace and Mathews, can be used to quantify disease progression in terms of beta-cell function, with various drug therapies. ${ }^{18}$

T2D is also characterised by the inevitable decrease in effectiveness of prescribed drugs, and a gradual increase in the intensity of glucoselowering therapy requirements. ${ }^{11,12}$ The necessity to intensify therapy by increasing doses of drugs, increasing frequency of dosage, or adding new drugs, can be taken as a marker of disease progression. While the number of oral drugs prescribed may be a discontinuous variable, insulin dose requirement is a continuous variable which lends itself to this indication. Disease progression in clinical trials in insulin can be assessed by the number of injections required, as well as by the total daily dose requirement. ${ }^{19}$

A simple way of assessing disease progression over time is to measure change in $\mathrm{HbA}_{1} \mathrm{C} .{ }^{10} \mathrm{~A}$ clinically significant change in $\mathrm{HbA} 1 \mathrm{C}$ (recommended as an increase by $\geq 0.5 \%$ ) using a particular therapy, suggests that beta-cell function has declined over time; this implies progression of disease, in spite of the prescribed therapy. ${ }^{20}$

Current discourse highlights the need for multifaceted, comprehensive management of diabetes, as opposed to a purely glucocentric one. This approach may extend to the definition of disease progression as well. Vascular complications tend to increase with advancing duration of T2D. Improvement in $\mathrm{HbA} 1 \mathrm{C}$, however, can reduce the risk of both macrovascular and microvascular complications. The progression of diabetes may be tracked by the surrogate marker of cardiovascular events. ${ }^{21}$ Occurrence of one or more cardiovascular events will imply worsening of T2D. Non-fatal myocardial infarction, non-fatal stroke, and hospitalisation for heart failure are the commonly accepted objective endpoints of cardiovascular progression.
In addition to cardiovascular outcomes, renal health is an integral part of T2D health. Renal outcomes have been the subject of exploratory analyses in earlier cardiovascular outcomes trials, but are now being prespecified as secondary outcomes. ${ }^{22}$ Significant fall in estimated glomerular filtration rate (eGFR), rise in serum creatinine, rise in albuminuria, and need for renal replacement therapy are well accepted objective endpoints of renal progression of disease. The same endpoints can be used to track disease progression of T2D as well. These signposts are especially important, as reliance on glycaemic markers such as HbA1c may be misleading in a situation where worsening renal function causes hypoglycaemia.

Diabetes retinopathy is a complication which can be diagnosed and staged objectively, with minimal inter-observer bias. This has been extensively used in large trials such as The Diabetes Control and Complications Trial (DCCT) and The Epidemiology of Diabetes Interventions and Complications (EDIC) to grade progression of disease..$^{23}$ New onset, or worsening of retinopathy, therefore, is an attractive candidate for defining disease progression in T2D.

\section{Comprehensive definition}

A comprehensive definition of disease progression would be one which can be used in a wide variety of patients, attending heterogeneous clinical care settings. We suggest the following: disease progression may be defined as gradual worsening of beta-cell function, clinically observed as an increase in drug dosage, drug frequency or number of glucose lowering drugs needed to maintain HbA1c control; and/or a $\geq 0.5 \%$ rise in HbA1C, unexplained by acute, modifiable factors, while using the same drug regimen; and/or as the occurrence or worsening of cardiovascular or microvascular complications, in spite of standard care, over a prespecified time period.

This definition includes pathophysiological, pharmacological and clinically relevant constructs and endpoints. It is easily understandable for both individuals living with T2D and their health care providers, and it can also be used across various healthcare settings, even if resource constraints prevent comprehensive monitoring of the disease progression. The objective aspect of this definition lends itself to measurement and comparison. Modern studies which offer insight into all aspects of this multifaceted definition of disease progression are needed. ${ }^{24,25}$

\section{Progression, outcomes and durability}

The last decade, we have witnessed more focus on clinical outcomes in T2D. This is evident from the heightened interest in cardiovascular outcome trials. ${ }^{21}$ While such trials are necessary, there is debate regarding their cost-effectiveness and relevance. The concept of disease progression differs from that of outcomes. While progression is dynamic, and is seen as a journey to an end, outcomes suggest a fatalistic approach to a fatal ending. Trials on disease progression study participants, while outcome trials, which are event-driven, tend to study events. Ideally, disease progression should be prevented before it reaches an irreversible stage. Outcome trials, on the other hand, enrol participants who are at high risk, in whom it is assumed that cardiovascular events are inevitable. The concept of prevention of disease progression, therefore, is a healthoriented one, which appeals more than the pathogenetic outcomeoriented approach. ${ }^{26}$

Disease progression is an overarching term which includes the concept of glucose lowering drug(s) durability. Pharmacological research treats 
durability of a drug as its capacity to maintain $\mathrm{HbA} 1 \mathrm{C}$ control over an extended period of time. ${ }^{27} \mathrm{~A}$ more comprehensive definition, however, is in order. We suggest that the durability of a drug, or a drug combination, be defined as its ability to postpone or delay progression of disease, in a safe and well-tolerated manner. Thus, all drugs which are able to postpone loss of glycaemic control, the need for intensification of therapy, and/or onset or worsening of complications, may be termed durable. For such an effect to take place and for a drug to be labelled as durable, we suggest 12 months' use as a minimum time frame. This choice can be explained by the fact that efficacy data relating to most anti-diabetic molecules is 12 months, and changes in therapeutic choice are suggested at 3-month intervals. ${ }^{28}$

\section{Summary}

The definition proposed for disease progression has been drafted with $\mathrm{T} 2 \mathrm{D}$ in mind, and does not extend to prediabetes. Most cases of prediabetes remain undiagnosed, and are evident only when they cross the threshold of T2D. Nevertheless, it is important to assess disease progression in this cohort as well, and this issue should be explored further. One of the main limitations of the proposed definition is that complications of T2D are multifactorial, and to attribute the same to failure of the anti-diabetic medication may be unwarranted. While we have included this in our definition to highlight the importance of thinking beyond $\mathrm{HDA} 1 \mathrm{C}$, we understand that this may not be relevant in all clinical situations.

Disease progression is not just a definition, but a framework for optimal strategy and clinically relevant goals. Delaying disease progression is not only a desired target, but a necessary strategy as well. To achieve this target, one must preferentially use glucose-lowering drugs which are proven to retard disease progression as well as the onset of complications in T2D. $\square$
. Remuzzi G, Ruggenenti P, Benigni A. Understanding the nature of renal disease progression. Kidney Int. 1997;51:2-15.

2. Leon BM, Maddox TM. Diabetes and cardiovascular disease: Epidemiology, biological mechanisms, treatment recommendations and future research. World I Diabetes. 2015;6:1246.

3. Zoja C, Zanchi C, Benigni A. Key pathways in renal disease progression of experimental diabetes. Nephrol Dial Transplant. 2015:30(supp|4):iv54-9.

4. DeFronzo RA. From the triumvirate to the ominous octet: a new paradigm for the treatment of type 2 diabetes mellitus. Diabetes. 2009;58:773-795

5. Kalra S, Chawla R, Madhu SV. The dirty dozen of diabetes. Indian J Endocrinol Metab. 2013;17:367.

6. Leslie RD, Palmer J, Schloot NC, Lernmark A. Diabetes at the crossroads: relevance of disease classification to pathophysiology and treatment. Diabetologia. 2016;59:13-20.

7. Babiker A, Mohammed Al Dubayee. Anti-diabetic medications: How to make a choice? Sudan J Paediatr. 2017;17:11-20.

8. Fonseca VA. Defining and characterizing the progression of type 2 diabetes. Diabetes care. 2009;32(suppl 2):S151-6. Nichols GA, Hillier TA, Brown JB. Progression from newly acquired impaired fasting glucose to type 2 diabetes. Diabetes Care 2007:30:228-33.

10. Pani LN, Nathan DM, Grant RW. Clinical predictors of disease progression and medication initiation in untreated patients with type 2 diabetes and $\mathrm{A} 1 \mathrm{C}<7 \%$. Diabetes Care. 2008;31:10.2337/dc07-1934.

11. Matthews DR, Cull CA, Stratton IM, et al. UKPDS 26: sulphonylurea failure in non-insulin-dependent diabetic patients over six years. UK Prospective Diabetes Study (UKPDS) Group. Diabet Med. 1998;15:297-303.

12. Kahn SE, Haffner SM, Heise MA, et al. Glycemic durability of rosiglitazone, metformin, or glyburide monotherapy. N Eng/J Med. 2006:355:2427-43.

13. Garber AJ, Abrahamson MJ, Barzilay Jl, et al. AACE/ACE comprehensive diabetes management algorithm 2015. Endocr Pract. 2015:21:438-47.

14. Zheng Y, Ley SH, Hu FB. Global aetiology and epidemiology of type 2 diabetes mellitus and its complications. Nat Rev Endocrinol. 2018;14:88.

15. Mathis $D$, Vence L, Benoist C. $\beta$-Cell death during progression to diabetes. Nature. 2001;414:792-8.

16. Kalra S, Gupta Y. Beta-cell insufficiency. Eur Endocrino 2017;13:51.

17. Palmer JP, Fleming GA, Greenbaum CJ, et al. C-peptide is the appropriate outcome measure for type 1 diabetes clinical trials to preserve $\beta$-cell function: report of an ADA workshop, 21-22 October 2001. Diabetes. 2004;53:250-64.

18. Wallace TM, Matthews DR. Coefficient of failure: a methodology for examining longitudinal beta-cell function in type 2 diabetes. Diabet Med. 2002:19:465-9.

19. Kalra S, Kalra B, Kumar S. The IMPERIAL study: physicians' perceptions regarding intensification of insulin therapy. Endocrine Abstracts. 2010:22:298.

20. Wangge, G, Michelle P, Mirjam J. Knol, et al. Regulatory scientific advice on non-inferiority drug trials. PLOS ONE. 2013;8:e74818.

21. Schnell O, Rydén L, Standl E, Ceriello A. Current perspectives on cardiovascular outcome trials in diabetes. Cardiovasc Diabetol. 2016;15:139
22. Rosenstock J, Perkovic V, Alexander JH, et al. Rationale, design, and baseline characteristics of the CArdiovascular safety (CARMELINA $\left.{ }^{(}\right)$: a randomized, double-blind, placebo-controlled clinical trial in patients with type 2 diabetes and high

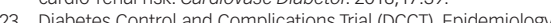
of Diabetes Interventions and Complications (EDIC) Research of Diabetes Interventions and Complications (EDC) Research Group, Lachin JM, et al. Effect of intensive diabetes therapy on the progression of diabetic retinopathy in patients with type diabetes: 18 years of follow-up in the DCCT/EDIC. Diabetes. 2015;64:631-42

24. Kalra S, Baruah MP, Sahay R, Kishor K. Pentads and hexads in diabetes care: numbers as targets; numbers as tools. Indian $J$ Endocr Metab. 2017;21:794-6.

25. Del Prato S, Foley JE, Kothny W, et al. Research: Treatment Study to determine the durability of glycaemic control with early treatment with a vildagliptin-metformin combination regimen vs. standard-of-care metformin monotherapy-the VERIFY trial: a randomized double-blind trial. Diabet Med. 2014:31:1178-84.

26. Kalra S, Baruh MP Sahay R. Salutogenesis in type 2 diabetes Kalra S, Baruah MP, Sahay R. Salutogenesis in type 2 diabetes 2018:22:169-72.

27. Esposito $K$, Chiodini P, Maiorino MI, et al. Glycaemic durability with dipeptidyl peptidase-4 inhibitors in type 2 diabetes: a systematic review and meta-analysis of long-term randomised controlled trials. BMJ open. 2014;4:e005442.

28. Stein, SA, Elizabeth ML, Stephen ND. A Review of the Efficacy and Safety of Oral Antidiabetic Drugs. Expert Opin Drug Saf. 2013;12:153-75 